\title{
Diagnostic Coding: A Novel Approach to Quality Assurance in Private Practice
}

\author{
Jessie Payne ${ }^{1}$, Sandiya Sathiyaseelan ${ }^{1}$, Maureen E Beran ${ }^{1}$, Glynda Hurley ${ }^{1}$ and Roy G Beran*1,2,3,4 \\ ${ }^{1}$ Strategic Health Evaluators, Sydney, New South Wales, Australia \\ ${ }^{2}$ Griffith University, Gold Coast and Brisbane, Australia
}

${ }^{3}$ Department of Neurology and South Western Clinical School, Liverpool Hospital and University of New South Wales, Australia

${ }^{4}$ Sechenov Moscow First State University, Moscow, Russia

*Corresponding author: Roy G Beran, Consultant Neurologist, who is the corresponding author and the relevant institution is Strategic Health Evaluators (SHE), C/- PO Box 598, Northbridge, NSW 1560, Australia.

Received Date: February 11, 2019

Published Date: February 22, 2019

\begin{abstract}
Quality Assurance (QA) measures in private practice require the collaborative effort of practitioners and their administrative staff and are designed to monitor and improve the efficiency and quality of clinical practices and patient care. The private research company 'Strategic Health Evaluators' (SHE) in Sydney, Australia, has developed a unique QA system based on diagnostic coding to monitor follow-up treatment for patients, report incidents and assess the appropriateness of current patient treatment. Concurrently, the system is also useful in creating a pool of potential subjects for clinical trials as patients are categorised within a database by their diagnosis. This paper reports the benefits of the application of diagnostic coding in the private practice environment and evaluates the extent to which the system has achieved its goal of quality assurance.
\end{abstract}

\section{Introduction}

Quality Assurance (QA) procedures are critical in clinical practice to ensure the highest standard of patient-oriented care [1]. To guarantee that QA measures are being conducted and adhered to by healthcare practitioners, efficient operation systems must be in place to ensure that a patient's health, wellbeing and care are monitored, long after the patient has left the waiting room [1]. These systems aim to provide the highest quality of patient care through incident reporting and care monitoring [2,3], to reduce the risk and recurrence of errors, such as patient loss to medication errors [4], follow up, and patient non-compliance with treatment [2]. Methods of providing such QA are usually only practiced in large institutions and are usually not adopted by single practitioner clinics [5]. In large institutions, where much QA is in place, there is usually a department within the hospital which assures responsibility for monitoring and is answerable to the hospital administration rather than the clinicians who deliver patient care [6].

Strategic Health Evaluators (SHE) in Sydney, Australia, is a private research company operating in conjunction with, and as an integral component of, a single clinician community-based neurological outpatient service. Due to the limited resources available, as a result of being a small, privately owned practice, the clinic has developed a unique system of diagnostic coding to assess patient specific parameters to help monitor with patient care that is directly comparable to that of the measures offered in larger institutions. This diagnostic coding system examines patient files and identifies patients by their diagnosis, allowing for the neurologist and all staff to monitor patient treatment, compliance, communications, content of medical records and results and serves as a safeguard to prevent against medication errors and other anomalies.

The clinic, which is the subject of this review, has instigated a number of QA measures, such as writing to the referring practitioners, should a patient fail to keep an appointment without notifying the clinic. As the clinician sees approximately 100 patients per week, it is important to both maintain QA as well as undertake clinic audits to ensure that the additional demands, imposed by QA procedures, are valid and productive. Diagnostic coding requires significant time investment, work delegation and collaboration of practice staff with the objective of verifying that treatment regimens were adhered to; that the risk of medication errors was minimised; that necessary tests have been performed; patient compliance was maintained with proper follow up ensured; 
and that correspondence between specialists, general practitioners and carers was complete.

Being a research company, the diagnostic coding system not only serves to provide a means for effective QA but also aids in the clinical trial recruitment process by identifying a possible source of trial candidates, found to have specific diagnoses. The purpose of this paper is to determine if the time, collaborative effort and resources, necessary to conduct diagnostic coding and QA, was of sufficient benefit to the practice and its patients to justify continuing the process, and, if so, to what extent has it satisfied the goal of QA.

\section{Method}

At SHE, patients who attend the practice are assigned a medical reference number (MRN), numerical in sequential order, similar to the procedure adopted in much larger institutions. All medical documents related to a patient are filed into that patient's medical record using their MRN as an identifier, as patient records are filed by number rather than alphabetically. During diagnostic coding, 50 recent patient files are selected and offered for review for every 50 60 new patients that are seen at the clinic. The files are selected based on MRN, in sequential lots of 50, leaving a gap of 100-150 files (MRN numbers) between the newest patient to the practice (who will have the highest MRN), and the first MRN in that set of diagnostic coding files. For example, if the newest patient's MRN is 2000, the diagnostic coding process will begin with MRN 1850 and end in MRN 1900, and after seeing 50 new patients (namely, the last seen will have MRN 2050), the next diagnostic coding set of 550 will begin with MRN 1900 and the last MRN will be 1950). The purpose of leaving this gap, between the latest patient and the start of the diagnostic coding set, is to allow for follow up material (such as letters and results) to be received by the practice and to allow for sufficient time to establish a better working diagnosis or for necessary tests to be performed. It also overcomes some logistic problems that may delay certain tests to be performed, such as in-patient polysomnography, as the clinician is also an accredited sleep physician.

Should a follow up letter be required, consequent to review of the patient's record, the neurologist will dictate such a letter, which will be sent to the relevant recipient- which may be a general practitioner, an authority such as the driver licensing authority, a caregiver or another referring doctor and, where appropriate, copied to the patient. Patients with particular medical conditions or diseases will have these diagnoses coded and the files noted by said diagnosis, for future reference. This confirms that the file has been reviewed and allows for ease of access, should research focus on that medical condition. If a patient's file needs to be accessed by the neurologist or a staff member, dependent on their diagnoses, the diagnosis is entered into the system and the MRN's of all patients with that diagnosis will appear.

Diagnostic coding requires specific time allocation - the neurologist will require 1.5 hours on average to review 50 files and complete any necessary dictations, or institute follow up procedures, whilst concurrently recording the diagnosis. The medical typist will require 2-3 hours to produce appropriate correspondence as required and to document diagnostically coded patients by their MRN with diagnostic categories. Responses from patients, healthcare specialists, caregivers and authorities are monitored by the neurologist and the office staff to ensure that all recommended follow up procedures are performed. The process requires a significant amount of invested time by all concerned.

If, at the time of coding, a patient's file is unavailable, due to the patient having an appointment and their file allocated for a consultation, diagnostic coding will need to occur following that consultation. This may necessitate extra time per patient depending on the complexity of the tasks involved, such as making a diagnosis, entering new codes electronically and preparing a follow up letter (if needed).

If action is required by the patient, as a consequence of the diagnostic coding having identified a deficiency, such as having a test performed before resuming driving, this is notified to the referring doctor, and with the correspondence copied to the patient. Office staff maintains a separate record of such correspondence to ensure compliance and, should there be no response, then follow up action, such as notifying driving authorities, ensues.

\section{Results}

Between the 1st of May 2013 and the 13th of April 2016, patient files 19951-21450 inclusive, and file 13842 were reviewed for diagnostic coding. Of the 1501 patient files reviewed, 1263 (84.1\%) required no further action after coding, whilst the remaining 238 patients (15.9\%) required a follow up letter. During our review, no medical errors or other anomalies were noted and no medication revisions were required. Thirty two of the 238 letters sent were regarding fitness to drive assessments; more than half (20/32) required further correspondence to the Roads and Maritime Services attesting a lack of fitness to drive, eight required no further action due to appropriate follow-up having taken place, and four patients were lost to follow up regarding driving fitness.

Thirty of the 238 letters regarded follow up of results, reminders or cancellations of Magnetic Resonance Imaging, Electroencephalograms, polysomnographs, and/or CPAP titrations, including 14 patients who did not attend their appointments, cancelled their appointments or failed to follow through with clinical advice. Correspondence was returned by the GP of one patient to inform the practice that the patient had since died. Ten letters indicated that the patient was under the care of another specialist and did not need to return to the practice. Other patients had failed to keep follow up appointments at the practice, failed to have necessary tests performed, had been ordered therapy with no indication of success (or otherwise) and left doubt that proper care had been provided. Many letters simply sought feedback to ensure that the patient was receiving appropriate care.

Three potential trial candidates were able to be identified by the trial coordinator using the diagnostic coding categories and were subsequently screened for inclusion into the suitable trials.

\section{Discussion}

\section{Neurologist's perspective}

The neurologist, being a sole practitioner, used the diagnostic coding process to ensure QA was undertaken within the 
community-based clinic and that patients, GPs and authorities were afforded best practice. It also allowed review of correspondence, in response to the original letters sent to referring practitioners, and to dictate further follow up as required. One benefit of this process, from the perspective of the neurologist, was that it reassures patients, carers, GP's and authorities of a commitment to QA and patient care. Despite not having the infrastructure that is available within larger institutions, this process ensured that there was a reliable contact, in terms of prompt and thorough correspondence, regarding patient care. It also provided the neurologist a window on the clinical referral base, the diagnoses of the patients seen, that tests were properly evaluated, and that treatment provided was effective. It enforced a review of correspondence sent, ensured referring doctors received necessary feedback and that necessary tests were performed, results received and reviewed and follow up management undertaken where necessary. It protected against situations where patients neither heeded advice, nor had necessary tests performed. It ensured that the neurologist met clinical, ethical and legal obligations which mirror the service provided in larger facilities but were not usually put in place in small single practitioner clinics.

\section{Typist's perspective}

Responsible for the transcription of the neurologist's findings, following the review process, the typist was required to spend 2-3 hours preparing and sending appropriate correspondence to patients, GPs, carers and authorities, and coding patient diagnoses into the diagnostic coding file. She was also responsible for making notes in the patient's medical file, if a response was received, if more correspondence and follow up care notifications were required, and to maintain a procedure for further follow up if needed, such as notifying licensing authorities. This required significant time delegation (approximately 3 hours) away from various other administrative duties, as well as ensuring that all files, within the 50 identified for scrutiny, were reviewed by the neurologist, particularly if they were not available, at the time of initial coding. This added an additional layer of responsibility for the typist but also contributed to job satisfaction within a very small administrative team and served to add value to the overall process.

\section{Clinical trial coordinator's perspective}

Diagnostic coding was beneficial to the clinical trial coordinator, in conjunction with the neurologist (who had the dual responsibility of conducting private care and acting as the principle investigator for clinical trials conducted by SHE), as it allowed for the utility of a diagnoses database; which serves to identify patients who may be eligible for inclusion within clinical trials. The diagnostic coding system only required the search of the key diagnoses, as stipulated within any given trial inclusion criteria, to nominate potential participants and a subsequent search of each identified patient's record to confirm co-morbidities which might fall under exclusion criteria, thereby rendering them either eligible or ineligible for any given trial. Considering that a large number of clinical trials do not achieve their recruitment goals [6], this proved advantageous to both the clinical trial coordinator and potential study participants in that a greater pool of participants could be chosen for screening and less time was required in ascertaining trial eligibility as the coordinator was able to review the patient's medical file prior to the screening appointment.

\section{Conclusion}

There were identified benefits attached to the diagnostic coding process to SHE staff, clinic staff, patients and other practitioners. For patients, follow-up correspondence encouraged them to comply with ongoing care. It motivated them to return, either to the practice for follow up consultations, or to seek suitable care elsewhere. It reaffirmed to the patient that the neurologist was committed to the best quality of care for them and strengthened the doctor-patient relationship which is considered so critical to QA in healthcare [7]. It may also ensure that any medication errors or other anomalies are quickly identified and corrected; thereby ensuring patients received the correct course of treatment. Whilst only 238 letters were required to be sent, within the 36-month period, QA could not be guaranteed for only 25 patients- 14 patients did not follow up with advice as requested, 10 attended a new practice and 1 passed away, providing strong evidence that the coding system, instigated by SHE, was an effective form of QA. Considering that a significant amount of time and effort was required, to execute the QA procedures adopted during the diagnostic coding process, it is reassuring that only 238 letters were required $(\sim 16 \%)$ which translated to $\sim 84 \%$ of files being correctly managed and optimal patient care being provided. It confirmed that patients were being properly managed, and lines of communication were being maintained between the consultant, referring practitioner and others involved in patient care, recognising that the subject of this study was a solo neurologist in a community based, and private practice clinic devoid of the large infrastructure available in large academic institutions. In addition, the organisation of patients by diagnosis provided the trial coordinator with a pool of potential clinical trial patients, thereby aiding in the recruitment process and resulting in efficient research within the clinic. Staff also felt more involved in the long-term process of patient care which contributed to job satisfaction. Considering these benefits and gains, as a consequence of the adopted practices, it was felt that the procedures did justify the time commitment and quality of care offered to patients. On this basis, it was felt that diagnostic coding was a worthwhile component of QA and should continue.

\section{Acknowledgement}

None.

\section{Conflict of Interest}

No conflict of interest.

\section{References}

1. Rao GN (2002) How Can We Improve Patient Care? Community Eye Health15(41): 1-3.

2. The RACGP Curriculum for Australian General Practice (2011) Quality and Safety- The systems approach to quality and safety.

3. Australian Commission on Safety and Quality in Health Care, National Safety and Quality Health Service Standards (September 2012). Sydney. ACSQHC 
4. Reason J (2000) Human error: models and management. BMJ 320(7237): 768-770.

5. Spath RG, Pickering KC, Webb SM (2003) Quality assurance and hospital structure: how the physician-hospital relationship affects quality measures. Ann Health Law 12(2): 235-247.
6. Black N (2010) Assessing the quality of hospitals. BMJ 34: 2066-c2066.

7. Longcope WT (2013) Doctor-Patient Relationship. Medicine: Preserving the Passion: 199. 